\title{
The Effectiveness of Cognitive Rehabilitation on Improving the Selective Attention in Patients with Mild Cognitive Impairment
}

\author{
Arezoo Shomali Oskoei ${ }^{{ }^{*}}$, Vahid Nejati ${ }^{2}$, Bita Ajilchi ${ }^{3}$ \\ ${ }^{1}$ Department of Psychology, Faculty of Human Science, Islamic Azad University of Rudehen (RIAU), Tehran, Iran \\ ${ }^{2}$ Department of Psychology, Faculty of Human Science, Shahid Beheshti University, Tehran, Iran \\ ${ }^{3}$ Department of Psychology, Faculty of Human Science, Science and Research Branch University, \\ Islamic Azad University (IAU), Tehran, Iran \\ Email: *Arezoooskoei@yahoo.com
}

Received August 28, 2013; revised September 27, 2013; accepted October 12, 2013

Copyright (c) 2013 Arezoo Shomali Oskoei et al. This is an open access article distributed under the Creative Commons Attribution License, which permits unrestricted use, distribution, and reproduction in any medium, provided the original work is properly cited.

\begin{abstract}
Purpose: The aim of the present study is to evaluate the effectiveness of cognitive rehabilitation on improving selective attention in patients with mild cognitive impairment. Methods: It was a quasi-experimental study with pre-test and posttest. The population in this study was all individuals referred to a neurology clinic in Tehran in 2012. The group was comprised of 40 patients with mild cognitive impairment who were evaluated with early detection and assessment by a medical psychologist (MMSE score lower than 25 and Wechsler memory test) and were selected by available sampling. They were also older than 55 years and had a minimum education at degree level, together with a lack of neurological and psychiatric comorbidities and impaired sensory and motor retardation, according to their nursing history and medical records. They were randomly divided into experimental and control groups (20 patients in each group). The experimental group was given 12 sessions (two hours each section) of cognitive rehabilitation with Neurocognitive Joyful Attentive Training Intervention (NEJATI). The control group, as expected with this group, did not receive any trial period. The selective attention of both groups was evaluated, before and after receiving intervention, by a Strop computer programme. Data were analysed using the covariance statistical test, MANCOVA. Results: The results show an increase in selective attention scores in the experimental group compared with the control group. Therefore, we can conclude that cognitive rehabilitation leads to improvement in the performance of selective attention ( $\mathrm{F}=4 / 97$; $\operatorname{sig}<0 / 05)$. Conclusion: Cognitive rehabilitation can impact on improving selective focus in people with mild cognitive impairment.
\end{abstract}

Keywords: Mild Cognitive Impairment; Cognitive Rehabilitation; Selective Attention

\section{Introduction}

Mild cognitive impairment is also called early dementia or discrete memory disorders; mild cognitive impairment indicates the cognitive deficit. Cognitive deficit can be considered as a disorder when it is below the expected level based on an individual's education and age, and interferes in people's everyday lives. The disorder affects many areas of thought and action such as language, attention, reasoning, judgment, reading and writing. Mild cognitive impairment in the most common form causes some memory problems [1]. The prevalence of mild cognitive impairment increases with age. Its prevalence among 70 - 79 years old people is about $10 \%$ and for

\footnotetext{
${ }^{*}$ Corresponding author.
}

80 - 89 years old people, about 25\%. Mild cognitive impairment is on a continuum between normal ageing and early signs of dementia. People with mild cognitive impairment do not work well on memory tests; however, they generally operate at normal levels. Almost half of the people who already have mild cognitive impairment will, 3 to 5 years later, be diagnosed with Alzheimer's disease [2]. Mild cognitive impairment is specified either by forgetfulness or with attention deficit. Data obtained from the patient's show that attention is affected early in the disease and the main impairment in implicit memory appears in association with selective attention deficit [3]. Selective attention is the ability to maintain the sources of attention on a specific stimulus and eliminate harmful stimuli. The highest level of attention is selective atten- 
tion which includes careful attention and response to stimuli [4]. Cognitive weaknesses are gradual during early adulthood. Although the ageing brain is involved in it, the brain can develop nerve fibers to compensate for them. In addition, some cognitive weaknesses may be due to the lack of specific skills, rather than biological ageing. If flexibility in growth is possible in ageing, the cognitive training intervention should to some extent reduce the cognitive weaknesses [5]. One of them is cognitive rehabilitation. Cognitive rehabilitation includes a wide range of therapeutic methods which are applied by different rehabilitation specialists. Many rehabilitation professionals may benefit from cognitive rehabilitation approaches to promote their clients' cognitive functions. In cognitive rehabilitation, the therapist records the data from the evaluation including the percentage of correct answers and the speed of the performance. If the participants master the task, the therapist increases the degree of difficulty of the task [6]. CARF (Commission on Accreditation of Rehabilitation Facilities, 1999) developed an operational definition of cognitive rehabilitation in which cognitive rehabilitation included therapeutic activities focused on performance that aimed to reinforce or re-establish old patterns, and establish new patterns of behaviour, or provide a mechanism to compensate for the cognitive functions of the damaged nervous system. In cognitive rehabilitation, we have two major approaches, the compensation or compromise approach that aims to remove individual restrictions by making changes in the environment, habits and way of doing things and administrative guidelines. These three goals of treatment remind us of Cognitive Ergonomics which is based on principles of information processing and reduce working memory involvement. The second approach, cognitive therapy, is an attempt to restore lost cognitive capacities by training and providing purposeful incentives; its aim is to improve individual performance. It should be noted that the separation of these two approaches will never happen in practice and their overlap in treatment is inevitable [7]. Different research carried out in relation to the cognitive training focused on the memory, attention, language and executive functions [8]. They have shown the possibility of recovery in these cognitive deficiencies [9], but a few studies have been carried out on rehabilitation of these deficiencies in older patients who have a moderate cognitive disorder [10]. Of course, positive results of these techniques have also been indicated in people with dementia, brain stroke and trauma [11]. In relation to the impact of cognitive rehabilitation on people with moderate cognitive disorder, it can be noted from the research of Talassi, Guerrreschi, Feriani, Fedi, Bianchetti, \& Trabucchi [12] that the recovery of the cognitive and emotional statuses can be also seen in these people. Furthermore, Kurz, Pohl, Ramsenthaler \& Sorg [13] observed the impact of cognitive rehabilitation in daily activities, mood and memory of these people in their own studies. Schmitter \& Edgecombe [14] also found in their studies that the cognitive rehabilitation navigations could create better results in the behaviour management skills of people with moderate cognitive impairment. Moreover, Stavros, Fotni and Magda [15] also found that the attention abilities and memory of people with this impairment can be recovered by cognitive rehabilitation. However, Huckans, Hutson, Jak, Kaye, Storzbach and Twamley [16] reported the necessity of a remedial approach based on the evidence of cognitive rehabilitation of people with moderate cognitive impairment in reaching a theoretical model of rehabilitation for developing and evaluating these therapeutic affairs in this case. As it has been shown, much research has been carried out on these issues to reach the most important consequences of the related evaluation such as cognitive function, life quality and intensity of neural symptoms. However, a few studies have been undertaken in relation to the evaluation of the impact of rehabilitation on changing the moderate cognitive impairment towards the natural cognition [16]. In addition, due to the reduction in the efficacy of the selective attention occurring for the cognitive impairment, it seems that upgrading the cognitive functions through cognitive rehabilitation can recover the deficiency of the attention. It also boosts the process of learning and the quality of daily life among these patients. The main aim of the present study is to review the effectiveness of the cognitive rehabilitation on the recovery of selective attention of people with moderate cognitive impairment and try to give a new approach in this case.

\section{Method}

The method used in this study is a quasi-experimental method with pre-test and post-test. The study population was all patients referred to a neurology clinic in 2012. 40 patients were selected, based on samples, and randomly divided into experimental and control groups. They were selected based on a diagnosis of mild cognitive impairment, the clinical diagnosis was made by a neurologist and psychiatrist based on an MMSE score lower than 25 and the Wechsler Memory Test. Control variables in this study were: the patients were older than 55 years and had a minimum education at degree level, a lack of neurological and psychiatric comorbidities, and impaired sensory and motor retardation, according to their nursing history and medical records. Both groups initially underwent selective attention assessment. The experimental group each received a cognitive rehabilitation period of 12 sessions twice a week for almost two and a half hours. The control group, as the excepted group, did not receive any trial period. After the intervention both groups were again assessed for selective attention. Finally, the data of 
30 subjects who successfully completed the rehabilitation period ( $\mathrm{n}=15$ Experimental group, 15 controls) were analysed by Mancova statistical tests.

\section{Instruments}

- Mini Mental State Examination: MMSE is a widely used screening test that was developed in 1975 by Flostein. Its purpose was to facilitate the differential diagnosis of patients in the hospital, but its main use is to assess the patient's cognitive abilities. This test is often referred to as a screening tool. "Mini Mental State Examination" is the most common screening tool for cognitive impairment in the world which has been translated and modified for different languages and cultures. The maximum score on this examination is 30 and a score below 25 suggests the possible demolition of cognition and a score below 20 indicates a definite demolition. In a cross-sectional study on the elderly people of Tehran, to determine the psychometric properties of a brief cognitive examination and prepare its normal tables, the validity of the test results were satisfactory (78/0) and a sensitivity of $90 \%$ and specificity of $84 \%$ in a cut-off point of 21 was obtained. The correlation among scores with age and education was significant at 0.05 levels [17].

- Wechsler Memory Scale (WMS-O): this is the most common memory test scale for adults. 10 years continual examination has been undertaken to develop its simplicity, speed and practicality. This scale was constructed and validated by David Wechsler in 1945 at a hospital in New York Blower. The Wechsler Memory Scale has two forms, A and B, each of which consists of seven different categories (general and personal information, orientation, mental control, and logical memory, repetition of digit, visual memory and learning associations). The Wechsler Memory Scale has been validated in the year 1993 by Sarrami. In the Sarrami study a sample size of 1007 people aged 64 20 was examined who were categorised into 9 groups. In this study Cronbach's alpha reliability test was 0.85 , and the results of internal consistency and factor analysis, validity and face validity were acceptable. The results obtained show Scale features in line with results of other studies [18].

- Stroop Color-word Test: This test is one of the most widely used tests of selective attention or focused attention and answer inhibition [19]. It is an experimental model as a basis for testing frontal lobe function. In the present study it was used in a computer type that comprised the following three steps. A) The first stage, which is a stage of coordinated efforts, the names of the four primary colours with black will appear in the centre of the computer screen and participants should press one of the red, yellow or green keys as soon as possible. B) Secondly, the names of four colours, each with their own colour appears in the centre of the computer screen and participants should press the appropriate key as soon as possible. C) The third stage is the stage of uncoordinated efforts or interferance in which the names of four colours, each with a different colour appears on the screen and participants should press the appropriate key as soon as possible. For example, the word red is written with another colour e.g. green and participants should determine the colour of the ink. Parameters measured in this test are: accuracy (number of correct answers) and speed (mean reaction time of correct responses to stimuli in milliseconds).The Stroop test is an in vitro model for the assessment of selective attention and it is a test of frontal lobe function. Reliability of the Stroop test, based on Otello and Graf's research [cited in 20] for three attempts are respectively equal to 0.76 , 0.83 and 0.97 .

- NEurocognitive Joyful Attentive Training Intervention (NEJATI): In the present study, training was used for enhancing selective attention via a cognitive rehabilitation computer programmes, were conducted in the meeting periodically. In each session four tasks were performed. The difficulty of the tasks increased stage by stage. If one stage finished in the expected time and conditions the next stage would be more difficult [21].

\section{Results}

As it can be seen in Table $\mathbf{1}$ the post-test mean of all variables are somewhat increased. In order to investigate the effect of rehabilitation on selective attention variables related to the real-time response and commission errors were measured before and after the experiment by multivariate covariance analysis. In this case, the pre-test scores of the two groups in reaction time and committing errors have the role of covariance variables and the post test scores have the role of dependent variables. Accordingly, the differences among the two groups in the post test were studied. The requirement of homogeneity was conducted according to the Box test. According to the

Table 1. Descriptive characteristics of selective attention in two groups before and after intervention.

\begin{tabular}{ccccc}
\hline Groups & Stage & Dependent Variables & Mean & SD \\
\hline Experimental & Pre-test & Reaction time & 7.70 & 5.82 \\
& & Commission error & 5.80 & 4.93 \\
& Post-test & Reaction time & 161.53 & 16.62 \\
& & Commission error & 147.46 & 13.67 \\
Control & \multirow{2}{*}{ Pre-test } & Reaction time & 8.93 & 7.84 \\
& & Commission error & 8.11 & 7.64 \\
& \multirow{2}{*}{ Post-test } & Reaction time & 168.80 & 22.02 \\
& & Commission error & 164.93 & 25.57 \\
\hline
\end{tabular}


Box's M (335.401) and F (2.041) obtained and significant probability (0.054), we can conclude that the data does not violate the variance-covariance matrix. The requirement of homogeneity of variances was also examined using a Levin test. According to the $\mathrm{F}$ values obtained the assumption of homogeneity of variance between the two variables was confirmed

According to Table 2 the Wilks Lambda value is more appropriate to report. The test results in the above table indicate that group has significant influence on the dependent variables $(P<0.05)$. Accordingly, we can say that after removing the effect of pre-test scores, in the linear combination of variables in the post-test some differences were observed between the two groups. Furthermore, based on discriminating factor coefficient we can say that about 25 percent of the variation in the linear combination of post test scores come from group which can be attributed to the experiment function.

The results of Table 3 show that after removing the effect of the pre-test a statistically significant difference exists in committing errors between the two groups $(\mathrm{P}<$ 0.05 ). Based on the discriminating factor coefficient presented in the last row we can say that in the committing error variable, $16 \%$ of the variance is caused by the group, which can be attributed to the experiment function. According to the table of means we can conclude that the mean of the experimental group is lower than that of the control group.

\section{Discussion}

The percentage of changes and the calculated size of selective attention in this research indicate a significant difference between the two groups. Thus, it can be argued that cognitive rehabilitation is effective in improving the performance of selective attention. These results are consistent with the results of many researchers [e.g.

Table 2. Multivariate tests.

\begin{tabular}{cccccc}
\hline Effect & Tests & Value & $\mathrm{F}(2,25)^{\mathrm{a}}$ & $\mathrm{P}<$ & Eta Square \\
\hline \multirow{3}{*}{ Group } & Pillai's Trace & 0.247 & 4.119 & 0.028 & 0.247 \\
& Wilks'Lambda & 0.752 & 4.119 & 0.028 & 0.247 \\
& Hotelling's & 0.329 & 4.119 & 0.028 & 0.247 \\
& Roy's Largest Root & 0.329 & 4.119 & 0.028 & 0.247 \\
\hline
\end{tabular}

${ }^{\mathrm{a}}$ Hypotesis $\mathrm{df}=2$, Error $\mathrm{df}=25$.

Table 3. Between subjects.

\begin{tabular}{cccccc}
\hline Resource & $\begin{array}{c}\text { Dependent } \\
\text { Variables }\end{array}$ & $\begin{array}{c}\text { Sum of } \\
\text { Squares }\end{array}$ & $\begin{array}{c}\text { Mean of } \\
\text { squares }\end{array}$ & $\mathrm{F}(1,26)$ & $\begin{array}{c}\text { Eta } \\
\text { Square }\end{array}$ \\
\hline Group & Reaction time & 8.547 & 8.547 & 2.84 & 0.01 \\
& Commission error & 982.596 & 982.596 & $4.97^{*}$ & 0.16 \\
\multirow{2}{*}{ Error } & Reaction time & 78.201 & 3.007 & & \\
& Commission error & 5136.96 & 197.575 & & \\
\hline
\end{tabular}

12,15,22-25]. These results are also in contrast with a few researches [e.g. 26-28]. The result that cognitive exercise can delay cognitive and functional decline in healthy older adults has a potential impressive application in MCI. Cognitive interventions may be useful for people with MCI, because these individuals have a high need for treatment and retain their ability to learn and to apply their new knowledge. If cognitive intervention is designed properly, we can improve cognitive function in people with MCI, reduce their disability, and anxiety of cognitive defects. Hence, an effective intervention programme promises significant benefits in terms of cognitive abilities and quality of life [22]. The results of the study indicate that cognitive rehabilitation interventions for individuals with mild cognitive impairment are effective in improving selective attention. It can be used as a stand-alone therapy or in combination with treatment methods in reducing the memory deficits in patients with mild cognitive impairment. These results suggest that cognitive rehabilitation may be effective in improving cognitive function in people with MCI. It may perhaps delay cognitive impairment and the start of inability. In recent years a growing number of studies have evaluated the effect of cognitive exercise on MCI. These studies have shown that exercise may have a beneficial effect on the scale of objective empirical knowledge [22]. This is a pilot study and is conducted for the first time in Iran. It should be done in different experimental designs and different age groups. Due to computer programme used in this project, we just selected people with minimal education; it would also be better to conduct research on people without education. Therefore, based on the findings, cognitive rehabilitation for people with mild cognitive impairment is necessary because it may become Dementia and Alzheimer's disease.

\section{REFERENCES}

[1] Mayo Foundation for Medical Education and Research, (MFMER) “Mild Cognitive Impairment,” 2011. http://www.mayoclinic.com/health/mild-cognitive-impair ment/DS00553

[2] H. S. Anderson and R. O. Kuljis, "Mild Cognitive Impairment," 2010.

http://emedicine.medscape.com/article/1136393-overview

[3] R. J. Perry and J. R. Hodges, "Attention and Executive Deficits in Alzheimer's Disease. A Critical Review,” Brain, Vol. 122, No. 3, 1999, pp. 383-404. http://dx.doi.org/10.1093/brain/122.3.383

[4] H. P. Marvin, "Remediation for Brain Injury and Neurological Illness,” Springer Science, New York, 2012. http://dx.doi.org/10.1007/978-1-4614-1975-4

[5] L. Burke, “Developmental Psychology,” Vol. II, Arasbaran, Tehran, 2007.

[6] M. M. Sohlberg and C. A. Mateer, "Cognitive Rehabilita- 
tion: An Integrative Neuropsychological Approach,” The Guilford Press, New York, 2001.

[7] D. Lee and C. A. Riccio, "Understanding and Implementing Cognitive Neuropsychological Retraining,” In: R. C. D’Amato, E. Fletcher-Janzen and C. R. Reynolds (Eds.), Handbook of School Neuropsychology, Wiley, Hoboken, 2005, pp. 701-720.

[8] T. A. Ala, L. G. Berck and A. M. Popovich, "Using the Telephone to Call for Help and Caregiver Awareness in Alzheimer Disease," Alzheimer Disease \& Associated Disorder, Vol. 19, No. 2, 2005, pp. 79-84.

[9] S. Salloway, S. Correia and S. Richardson, "Key Lessons Learned from Short-Term Treatment Trials of Cholinesterase Inhibitors for Amnestic MCI," International Psychogeriatry, Vol. 20, No. 1, 2008, pp. 40-46. http://dx.doi.org/10.1097/01.wad.0000165509.45123.57

[10] E. C. Miotto, V. T. Serrao, G. B. Guerra, M. C. S. Lucia and M. Scaff, "Cognitive Rehabilitation of Neuropsychological Deficits and Mild Cognitive Impairment: A Review of the Literature,” Dementia \& Neuropsychologia, Vol. 2, No. 2, 2008, pp. 139-145.

[11] M. Amini, B. Dowlatshahi, A. Dadkhah and M. Lotfi, "Cognitive Rehabilitation of Cognitive Deficits in Elderly Patients with Alzheimer's Dementia," Iranian Journal of Aging, Vol. 5, No. 15, 2010, pp. 78-86.

[12] E. Talassi, M. Guerrreschi, M. Feriani, V. Fedi, A. Bianchetti and M. Trabucchi, "Effectiveness of a Cognitive Rehabilitation Program in Mild Dementia (MD) and Mild Cognitive Impairment (MCI): A Case Control Study,” Archives of Gerontology and Geriatrics, Vol. 1, 2007, pp. 391-399. http://dx.doi.org/10.1016/j.archger.2007.01.055

[13] A. Kurz, C. Pohl, M. Ramsenthaler and C. Sorg, "Cognitive Rehabilitation in Patients with Mild Cognitive Impairment," International Journal of Geriatric Psychiatry, Vol. 24, No. 2, 2009, pp. 163-168. http://dx.doi.org/10.1002/gps.2086

[14] M. Schmitter-Edgecombe, “A Multi-Dyad Cognitive Rehabilitation Intervention,” 2010. http://www.alz.org/research/alzheimers_grants/for_resear chers

[15] Z. Stavros, K. Fotni and T. Magda, "Computer Based Cognitive Training for Patients with Mild Cognitive Impairment (MCI)," 3rd International Conference on Pervasive Technologies Related to Assistive Environments, New York, 2010. http://dx.doi.org/10.1145/1839294.1839319

[16] M. Huckans, L. Hutson, E. Twamley, A. Jak, J. Kaye and D. Storzbach, "Efficacy of Cognitive Rehabilitation Therapies for Mild Cognitive Impairment (MCI) in Older Adults: Working toward a Theoretical Model and Evidence-Based Interventions,” Neuropsychology Review, Vol. 23, No. 1, 2013, pp. 63-80.

[17] M. Foroughan, S. Jafari,, C. Shirin Baian, Z. Ghae Maqam Farahani and M. Rahgozar, "A Brief Examination of Standardized Cognitive Status of Elderly in Tehran,” Journal of New Cognitive Science, Vol. 38, No. 2, 2008, pp.
29-37.

[18] Gh. Sarrami, "Standardization of Wechsler Memory Scale (WMS-O) on the Population of Tehran," Master Thesis, Tarbiat Modares University of Tehran, Tehran, 1993.

[19] V. P. Bozikas, M. H. Kosmidis, G. Kiosseoglou and A. Karavatos, "Neuropsychological Profile of Cognitively Impaired Patients with Schizophrenia," Comperhensive Psychiatry, Vol. 4, No. 2, 2006, pp. 136-143. http://dx.doi.org/10.1016/j.comppsych.2005.05.002

[20] A. Karimi Ali Abad, S. M. Tamjid Kafi and H. Farahani "The Study of Executive Functions at Patients with Bipolar Disorders,” Advances in Cognitive Sciences, Vol. 12, No. 2, 2010, pp. 29-39.

[21] V. Nejati, H. R. Pouretemad and H. Bahrami, “Attention Training in Rehabilitation of Children with Developmental Stuttering,” Neuro Rehabilitation, Vol. 32, 2013, pp. 297-303. http://dx.doi.org/10.3233/NRE-130847

[22] S. Belleville, H. Chetrkow and S. Gauthier, "Working Memory and Control of Attention in Persons with Alzheimer's Disease and Mild Cognitive Impairment,” Neuropsychology, Vol. 21, 2007, pp. 456-469. http://dx.doi.org/10.1037/0894-4105.21.4.458

[23] V. K. Günther, P. Schafer, B. J. Holzner and G. W. Kemmler, "Long-Term Improvements in Cognitive Performance through Computer-Assisted Cognitive Training: A Pilot Study in a Residential Home for Older People," Aging and Mental Health, Vol. 7, No. 3, 2003, pp. 200206. http://dx.doi.org/10.1080/1360786031000101175

[24] G. Cipriani, A. Bianchetti and M. Trabucchi, "Outcomes of a Computer-Based Cognitive Rehabilitation Program on Alzheimer's Disease Patients Compared with Those on Patients Affected by Mild Cognitive Impairment (MCI): A Case Control Study," Archives of Gerontology and Geriatry, Vol. 43, No. 3, 2006, pp. 327-335. http://dx.doi.org/10.1016/j.archger.2005.12.003

[25] L. Rozzini, D. Costardi, B. Vicini Chilovi, S. Franzoni, M. Trabucchi and A. Padovani, "Efficacy of Cognitive Rehabilitation in Patients with Mild Cognitive Impairment Treated with Cholinesterase Inhibitors,” International Journal of Geriatric Psychiatry, Vol. 22, No. 4, 2007, pp. 356-360. http://dx.doi.org/10.1002/gps.1681

[26] J. O. Brooks, L. Friedman, A. M. Pearman, C. Gray and J. A. Yesavage, "Mnemonic Training in Older Adults: Effects of Age, Length of Training, and Type of Cognitive Pre Training," International Psychogeriatrics, Vol. 11, No. 1, 1999, pp. 75-84. http://dx.doi.org/10.1017/S1041610299005608

[27] L. Clare, R. T. Woods, E. D. Moniz, M. Cook Orrell and A. Spector, "Cognitive Rehabilitation and Cognitive Training for Early-Stage Alzheimer's Disease and Vascular Dementia (Cochrane Review),” No. 2, The Cochrane Library, Wiley, 2005.

[28] S. Rapp, G. Brenes and A. P. Marsh, "Memory Enhancement Training for Older Adults with Mild Cognitive Impairment: A Preliminary Study,” Aging and Mental Health, Vol. 6, No. 1, 2002, pp. 5-11.

http://dx.doi.org/10.1080/13607860120101077 